\title{
Berry-phase gates for fast and robust control of atomic clock states
}

\author{
Yunheung Song, ${ }^{1}$ Jongseok Lim $\odot,{ }^{2}$ and Jaewook Ahn $\circledast^{1, *}$ \\ ${ }^{1}$ Department of Physics, KAIST, Daejeon 34141, Korea \\ ${ }^{2}$ Centre for Cold Matter, Blackett Laboratory, Imperial College London, Prince Consort Road, London SW7 2AZ, United Kingdom
}

(Received 23 July 2019; revised manuscript received 15 January 2020; accepted 19 March 2020; published 16 April 2020)

\begin{abstract}
We propose and experimentally demonstrate a fast Berry-phase gate, which is implemented by picosecondtimescale optical pulses to make the qubit system of atomic clock states adiabatically evolve on a closed loop. The characteristic features of the proposed gate are gate speed and robustness against control fluctuations, which can potentially resolve the decoherence and reliability issues in quantum information processing, at the same time. The experiment is conducted with two linearly polarized, chirped optical pulses, interacting with five single rubidium atoms simultaneously in an array of optical tweezer dipole traps, to demonstrate the proposed picosecond-timescale clock-state gates. The robustness of the qubit rotation angle $\delta \Theta / \delta \mathcal{A}=1.5 \%$ is achieved with respect to the laser intensity (of pulse area $\mathcal{A}$ ) fluctuation.
\end{abstract}

DOI: 10.1103/PhysRevResearch.2.023045

\section{INTRODUCTION}

The Berry phase is one of the hallmarks of quantum mechanics, dealing with the geometric phase, gained by a quantum wave function subjected to an adiabatic process, which can remain nonzero even after a cyclic evolution in which the more familiar dynamic phase disappears [1]. It appears ubiquitously in numerous physical phenomena including the Aharonov-Bohm effect, the quantum Hall effect, and neutron interferometry, to list a few [1-3]. The Berry phase written as a unitary operator $U$ for a cyclic evolution is holonomy, which depends only on the evolution path but not on other dynamic details during the evolution. Thus, a geometrical manipulation of two-state systems utilizing the Berry phase is expected for robust quantum information processing against environment and parameter noises (characteristically of local nature) due to their independence from local phase changes (dynamic phases).

One way to implement these holonomic quantum gates $[4,5]$ is adiabatic time evolution $[1,6]$. The time evolution of a qubit system $\left|\psi\left(t_{i}\right)\right\rangle=\alpha|0\rangle+\beta|1\rangle$ driven by the time-varying field of the Hamiltonian $H_{I}(t)$ from $t_{i}$ to $t_{f}$ is written in the bare basis as

$$
\left|\psi\left(t_{f}\right)\right\rangle=e^{i \phi_{\mathrm{d}}} U(\Theta)\left|\psi\left(t_{i}\right)\right\rangle,
$$

where $\phi_{\mathrm{d}}$ is the dynamic phase that is only global, thus ignorable, and $\Theta$ is the geometric phase. The adiabatic time evolution of a qubit system allows no leakage from the initial adiabatic state of degenerate eigenenergy, so if an

\footnotetext{
*jwahn@kaist.ac.kr

Published by the American Physical Society under the terms of the Creative Commons Attribution 4.0 International license. Further distribution of this work must maintain attribution to the author(s) and the published article's title, journal citation, and DOI.
}

appropriate interaction picture removes this eigenenergy, a parallel transport condition $\left\langle\psi(t)\left|H_{I}(t)\right| \psi(t)\right\rangle=0$ can be imposed for the holonomy. Original proposals for holonomic quantum gates are based on this adiabatic evolution [4,79]. However, in many physical systems of limited coherence time, it is difficult to satisfy the adiabatic condition and experimental implementation has been limited to longlived transitions [10] or the shortcut to adiabaticity $[11,12]$. Most other examples utilize nonadiabatic holonomic quantum gates [13-17], but their nonadiabatic characteristic makes them sensitive to parameter fluctuations [18].

In the present paper we propose a method implementing adiabatic holonomic gates, thus Berry-phase gates, of atomic clock states in picosecond timescales. Experimental demonstration is performed on the hyperfine states of an atomic system, interacting with chirped optical pulses that allow adiabatic time evolution in the qubit system, to achieve the robustness of the Rabi oscillation angle $\delta \Theta / \delta \mathcal{A}=1.5 \%$ with respect to the laser intensity (pulse area) fluctuation. We further demonstrate a scheme for rotation operators about arbitrary axes, with which a set of universal one-qubit quantum gates can be constructed.

\section{THEORETICAL CONSIDERATION}

Let us consider an atomic system [see Fig. 1(a)], of an excited level $|e\rangle=\left|P_{1 / 2}\right\rangle$ and a ground level $|g\rangle=\left|S_{1 / 2}\right\rangle$, in which $|g\rangle$ consists of two ground hyperfine states (qubit states) $|0\rangle=\left|S_{1 / 2}, F=I+1 / 2, m_{F}=0\right\rangle$ and $|1\rangle=\mid S_{1 / 2}, F=I-$ $\left.1 / 2, m_{F}=0\right\rangle$. When the hyperfine energy splitting $\hbar \omega_{\mathrm{hf}}$ is negligible compared to the inverse of the gate-operation time, these qubit states can be considered as energy degenerate states.

We utilize a cyclic time evolution of the qubit system, by a pair of chirped pulses [see Fig. 1(b)]. As each chirped pulse implements a rapid adiabatic passage (RAP) [19] that provides robust adiabatic population transfer between $|g\rangle$ and $|e\rangle$, 
(a)

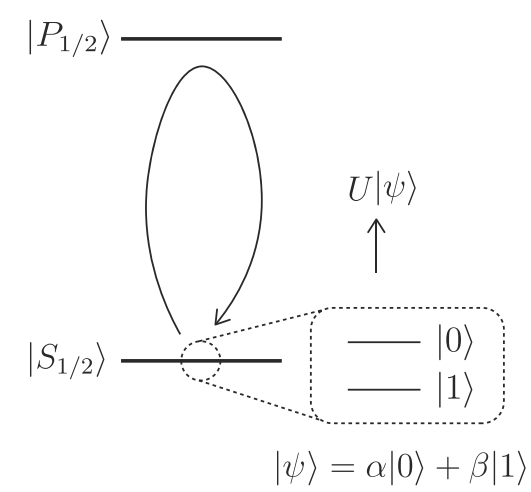

(b)

(i)

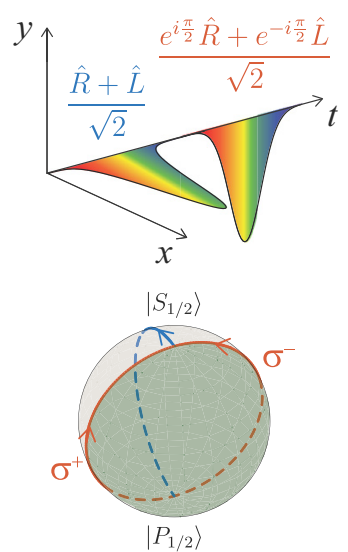

(ii)
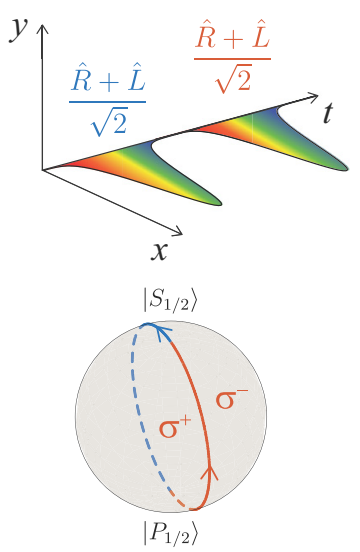

(iii)

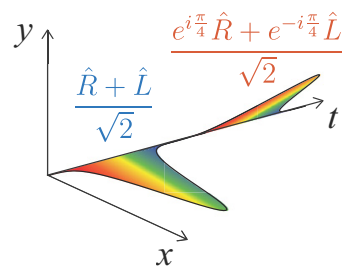

$\left|S_{1 / 2}\right\rangle$

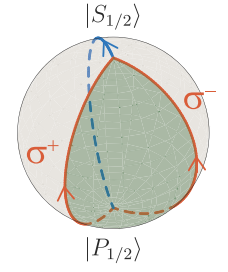

FIG. 1. (a) Energy-level diagram of a ground level $|g\rangle$, an excited level $|e\rangle$, and two degenerate qubit eigenstates $|0\rangle$ and $|1\rangle$ within the ground level $|g\rangle$. A cyclic transition between $|g\rangle$ and $|e\rangle$ results in the evolution from the initial qubit state $|\psi\rangle$ to $U|\psi\rangle$. (b) Pulse sequence of Berry-phase gates (top) and the corresponding time evolutions of the two-level system (bottom). When the system undergoes successive adiabatic passages by two linearly polarized, chirped laser pulses with relative polarization angle $\theta$ [(i) $\theta=-\pi / 2$, (ii) $\theta=0$, and (iii) $\theta=$ $\pi / 4]$, the first RAP excites the system from $|g\rangle$ to $|e\rangle$ along the path shown by the blue line and then the second RAP deexcites the system back to $|g\rangle$ along the two different paths labeled by $\sigma^{+}$and $\sigma^{-}$, shown by red lines. The geometric phase gained through the cyclic transitions is proportional to the shaded area enclosed by the two red lines in the Bloch sphere.

a pair of RAP applications adiabatically drives the transition from the ground initial state $\left|\psi\left(t_{i}\right)\right\rangle=\alpha|0\rangle+\beta|1\rangle$ to $|e\rangle$ by the first pulse and then back to the ground state by the second. Suppose the pulses propagate along the quantization axis $(+\hat{z}$ axis) of the qubit states $|0\rangle$ and $|1\rangle$, being linearly polarized with a relative polarization angle $\theta$ between them. Here we set the coordinate system to let the polarization unit vector of the first pulse be the $\hat{x}$-axis unit vector $\hat{x}=(\hat{R}+\hat{L}) / \sqrt{2}$, where $\hat{R}$ and $\hat{L}$ are the right and left circular polarization unit vectors, respectively. Then the polarization unit vector of the second pulse is expressed as $\left(e^{-i \theta} \hat{R}+e^{i \theta} \hat{L}\right) / \sqrt{2}$. Correspondingly, the qubit system is considered in the Cartesian basis, as $\left|\psi\left(t_{i}\right)\right\rangle=\frac{\alpha-\beta}{\sqrt{2}}|-\rangle+\frac{\alpha+\beta}{\sqrt{2}}|\rangle+$, where $| \pm\rangle \equiv(|0\rangle \pm|1\rangle) / \sqrt{2}$ are the fine-structure states $\left|S_{1 / 2}, m_{J}= \pm 1 / 2\right\rangle$ in our case. By the dipole selection rule, the right and left circular polarizations drive $\sigma^{ \pm}$transitions between $|\mp\rangle$ and $|e\rangle$, respectively.

The Bloch sphere representation in the lower figures of Fig. 1(b) shows the time evolution pathways of $|\mp\rangle$ driven by respective polarization components. After the cyclic evolution by the two pulses, $|\mp\rangle$ states get geometric phases $\pm \theta-\pi$, respectively, corresponding to $-\frac{1}{2}$ of the solid angle enclosed by the evolution pathways [5], while the dynamic phase $\phi_{\mathrm{d}}$ is due to the intensity- and detuning-dependent eigenenergy [19] and dynamic Stark shift from neighboring transitions [20]. Thus we get $\left|\psi\left(t_{f}\right)\right\rangle=\frac{\alpha-\beta}{\sqrt{2}} e^{i \phi_{-}}|-\rangle+\frac{\alpha+\beta}{\sqrt{2}} e^{i \phi_{+}}|\rangle+$, in which $\phi_{\mp}= \pm \theta-\pi+\phi_{\mathrm{d}}$ are the phases gained during the time evolution (see the Appendix for more details). The final state $\left|\psi\left(t_{f}\right)\right\rangle$ is then expressed in the qubit basis as

$$
\begin{aligned}
\left|\psi\left(t_{f}\right)\right\rangle & =-e^{i \phi_{\mathrm{d}}}\left(\begin{array}{cc}
\cos \theta & -i \sin \theta \\
-i \sin \theta & \cos \theta
\end{array}\right)\left|\psi\left(t_{i}\right)\right\rangle \\
& =-e^{i \phi_{\mathrm{d}}} U_{\hat{x}}(2 \theta)\left|\psi\left(t_{i}\right)\right\rangle,
\end{aligned}
$$

where $U_{\hat{x}}(\Theta)$ is the $X$-rotation operator of the qubit states by the rotation angle $\Theta=2 \theta$. Note that the dynamic phase $\phi_{\mathrm{d}}$ is always global because linear polarization guarantees an equal magnitude of the $\sigma^{ \pm}$transitions and thus the same dynamic phase for each transition. Therefore, this scheme implements the holonomic transition determined by only the geometric phase $\Theta$, robust against laser parameters such as intensity and detuning.

Qubit rotations about an arbitrary axis $\hat{n}=n_{x} \hat{x}+n_{y} \hat{y}$ can be implemented with an additional pair of time-delayed pulses. Since our scheme works in the regime where the hyperfine splitting is neglected, we adopt a method utilizing the hyperfine interaction in a longer timescale [20]. In the interaction picture where the qubit basis is $\left|0^{\prime}\right\rangle=|0\rangle$ and $\left|1^{\prime}\right\rangle=e^{-i \omega_{\mathrm{hf}} t}|1\rangle$, the Cartesian basis is given by $| \pm\rangle=\left|0^{\prime}\right\rangle \pm$ $e^{i \omega_{\mathrm{hif}} t}\left|1^{\prime}\right\rangle$. Then, with the second Berry-phase gate applied after time delay $T$, the time evolution of the qubit system from $t_{i}+$ $T$ to $t_{f}+T$ becomes $\left|\psi\left(t_{f}+T\right)\right\rangle=-e^{i \phi_{\mathrm{d}}} U_{\hat{n}}(\Theta)\left|\psi\left(t_{i}+T\right)\right\rangle$, where

$$
U_{\hat{n}}(\Theta)=\left(\begin{array}{cc}
\cos \theta & -i\left(n_{x}+i n_{y}\right) \sin \theta \\
-i\left(n_{x}-i n_{y}\right) \sin \theta & \cos \theta
\end{array}\right)
$$

is the rotation operator of the qubit states about the axis $\hat{n}$, with $n_{x}=\cos \left(\omega_{\mathrm{hf}} T\right)$ and $n_{y}=\sin \left(\omega_{\mathrm{hf}} T\right)$ controlled by the time delay $T$.

\section{EXPERIMENTAL PROCEDURE}

An experimental demonstration of the Berry-phase gates of atomic clock states was performed with an array of single rubidium atoms driven by pulse-shaped ultrafast optical pulses [see Fig. 2(a)]. Fast optical control of atomic and ion systems has been studied before through direct or Raman excitations [21-23], which we borrowed in the present work to implement adiabatic operations for geometric phases. Laser pulses were produced by a femtosecond Ti:sapphire amplifier system operated at a $1-\mathrm{kHz}$ repetition rate (carrier frequency $377.1 \mathrm{THz}$, bandwidth $3.8 \mathrm{THz}$ ), which were resonant to the $D_{1}$ transition. The pulses were linearly chirped by an 


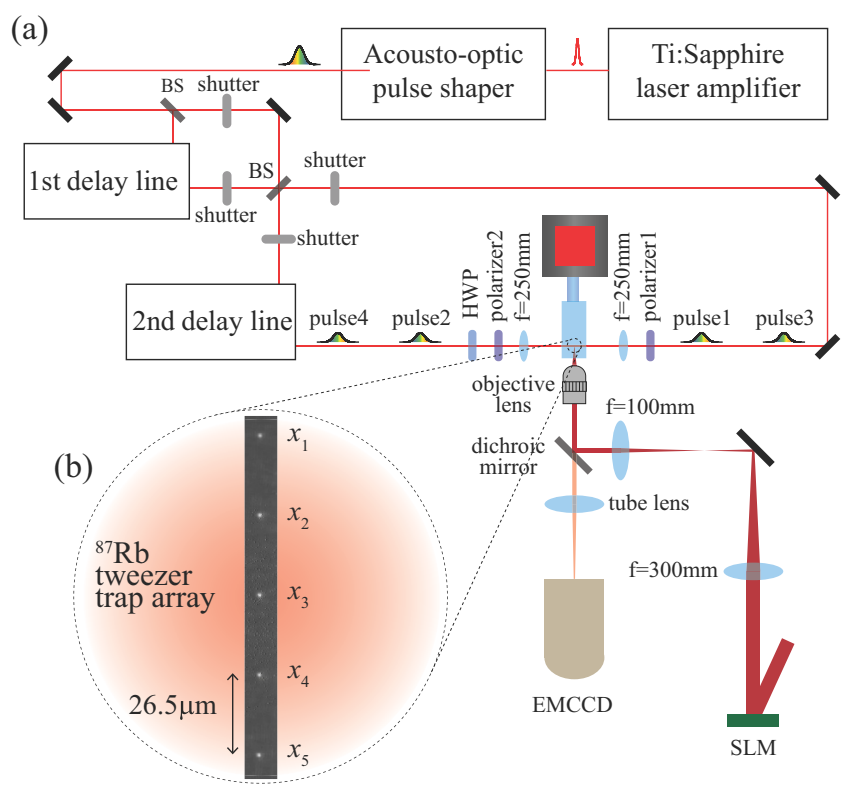

FIG. 2. (a) Schematic of the experiment. (b) Fluorescence image of single atoms at positions $x_{i}(i=1,2, \ldots, 5)$. The following denotations are used: BS, beam splitter; HWP, half-wavelength plate; EMCCD, electron multiplying charge-coupled device; and SLM, spatial light modulator.

acousto-optic pulse shaper to stretch the pulse length to $1.5 \mathrm{ps}$ with a chirp rate of $2.6 \mathrm{ps}^{-2}$, to satisfy the adiabatic condition for the RAP (see the Appendix for more details). Each pulse was split into two pairs of double pulses, with the interpair (intrapair) delay $T=70-370 \mathrm{ps}(\tau=6.7 \mathrm{ps})$. The relative polarization angle $\theta$ was varied by a combination of a half waveplate and a polarizer, realizing $U_{\hat{x}}$ with the first pair and $U_{\hat{n}}$ with the second. These pulses were delivered along the counterpropagating directions $( \pm \hat{z})$, respectively, to the atom array in a magneto-optical trap.

Five single atoms $\left({ }^{87} \mathrm{Rb}\right)$ were prepared by optical tweezers $[24,25]$ at fixed positions of $26.5-\mu \mathrm{m}$ spacing along the transverse direction of the laser beam propagation so that they experienced different intensities of the same laser pulses [see Fig. 2(b)]. The optical tweezers were tightly focused $852-\mathrm{nm}$ laser beams $\left(2-\mu \mathrm{m} 1 / e^{2}\right.$ diameter $)$ with a trap depth of $1.6 \mathrm{mK}$. The atoms were first optically pumped to the $|0\rangle=\left|5 S_{1 / 2}, F=2, m_{F}=0\right\rangle$ qubit state using $\pi$-polarized continuous light resonant with the $F=2 \rightarrow F^{\prime}=2$ transition of the $D_{1}$ line and the $F=1 \rightarrow F^{\prime}=2$ transition of the $D_{2}$ line, in the presence of an applied magnetic field of $2.4 \mathrm{G}$ which defined the quantization axis along the laser propagation axis.

Then the laser pulse sequence, each pair of which constituted one Berry-phase gate operation, was focused to the single-atom array with a beam waist of $60 \mu \mathrm{m}(90 \mu \mathrm{m})$ for pulses 1 and 3 (pulses 2 and 4) which was smaller than the array size of $106 \mu \mathrm{m}$. Thus each atom in the array experienced largely different laser intensities. Finally, a push-out measurement [26] was applied to record the probability of the $|1\rangle=\left|5 S_{1 / 2}, F=1, m_{F}=0\right\rangle$ state of each atom with an electron multiplying charge-coupled device camera.

\section{RESULTS AND DISCUSSION}

With the experimental apparatus, we first demonstrate the robustness of $U_{\hat{x}}(\Theta)$ in Eq. (2) against laser power fluctuation. We used the first pair of pulses [pulses 1 and 2 in Fig. 2(a)], while blocking the second pair (pulses 3 and 4), and measured the state $|1\rangle$ probability of each atom as a function of the relative polarization angle $\theta$. In Fig. 3(a) the measured probabilities $P(\theta)=\left|\left\langle 1 \mid \psi\left(t_{f}\right)\right\rangle\right|^{2}$ of the five atoms exposed to different position-dependent pulse areas $\left[\mathcal{A}_{\max } \approx 5 \times \mathcal{A}_{\min }\right.$; see Fig. 3(b)] are plotted and numerically fitted to the function

$$
P(\theta)=\gamma \sin ^{2}(\theta+\Delta \theta)+\eta,
$$

with fitting parameters $\gamma, \Delta \theta$, and $\eta$.

The ideal case is $\gamma=1$ and $\Delta \theta=\eta=0$, while experimental imperfection results in degraded fringe visibility $(\gamma<$ 1 and $\eta>0)$ and a fringe shift $(\Delta \theta \neq 0)$. Imperfections in $\gamma$ and $\eta$ that result in gate infidelities are due to errors in state preparation and measurement (SPAM). In our experiment, there exist optical pumping infidelity ( $\sim \%)$, push-out measurement infidelity $(\sim 3 \%)$, and polarization mismatch between the pulses and the quantization axis $(\sim 1 \%)$, in addition to the effect of weak pre- and postpulses [27]. However, we note that the SPAM errors are not directly related to the robustness of the Berry-phase gate.

On the other hand, a nonzero fringe shift $\Delta \theta \neq 0$ could imply failure of the intensity robustness of the proposed Berryphase gate. This error mainly came from the birefringence of the vacuum window [28], which affected the polarization of the laser pulses and the imbalance between the $\sigma^{ \pm}$transitions causing a dynamic phase error. This polarization imperfection was verified by measuring the gradient of the fringe shift $\delta \theta / \delta \mathcal{A}$ vs polarization ellipticity, as shown in Fig. 3(c). In our present demonstration of the $U_{\hat{x}}$ which was limited by the remaining polarization ellipticity of $1 / 40$, a robustness against the laser intensity is achieved up to $\delta \Theta / \delta \mathcal{A}=2 \delta \theta / \delta \mathcal{A}=$ $1.5 \%$, and the ultralow birefringence technique $[29,30]$ of $1 / 3000$ ellipticity is expected to further improve this below $\delta \Theta / \delta \mathcal{A}=0.01 \%$.

In the second experiment, we tested the robustness of the rotational axis $\hat{n}$, using two Berry-phase gates $U_{\hat{x}}$ (pulses 1 and 2) and $U_{\hat{n}}$ (pulses 3 and 4). The relative polarization angle $\theta$ of both gates was fixed to $\pi / 4$ for maximum visibility and the Ramsey fringe of the $F=1$ state probability $P(\theta, T)=$ $\left|\left\langle 1\left|U_{\hat{n}(T)}(2 \theta) U_{\hat{x}}(2 \theta)\right| 0\right\rangle\right|^{2}$ was measured, with respect to the time delay $T$ between the pulse pairs, and numerically fitted to the function

$$
P(\theta, T)=\gamma_{R} \sin (2 \theta) \cos ^{2}\left(\varphi_{\hat{n}}+\varphi_{0}\right)+\eta_{R},
$$

where $\gamma_{R}$ is the fringe visibility, $\varphi_{\hat{n}}=\omega_{R} T / 2$ is the angle of the rotational axis, $\varphi_{0}$ is the Ramsey phase shift, and $\eta_{R}$ is the offset. At each atom, the measured frequency resulted in $\left\langle\omega_{R}\right\rangle=(6.79 \pm 0.08) \times 2 \pi \mathrm{GHz}$, agreeing well with the ${ }^{87} \mathrm{Rb}$ hyperfine frequency within the $95 \%$ confidence interval, in which the equivalent time-domain error is as small as $0.9 \pm 1.8 \mathrm{ps}$.

In order to estimate how robust the rotation axis of the Berry-phase gate is, we consider the possibility of an intensity-dependent axis shift $\varphi_{0}(\mathcal{A})$, i.e., $\hat{n}(T) \rightarrow \hat{n}^{\prime}(T, \mathcal{A})$ 
(a)

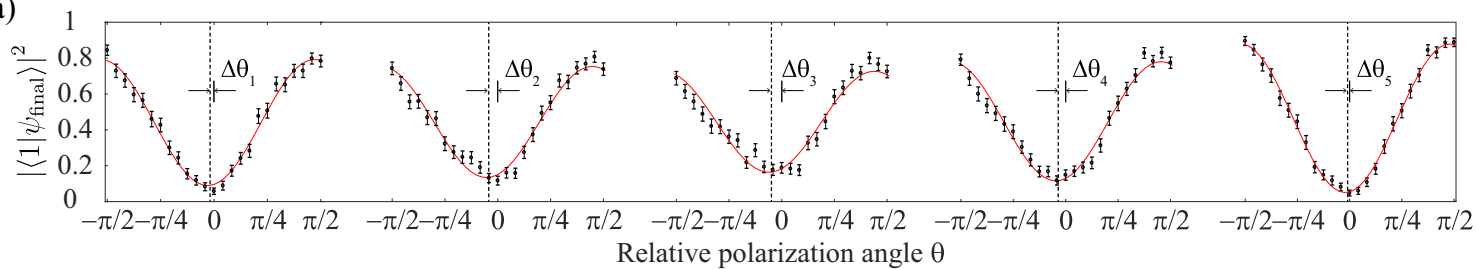

(b)

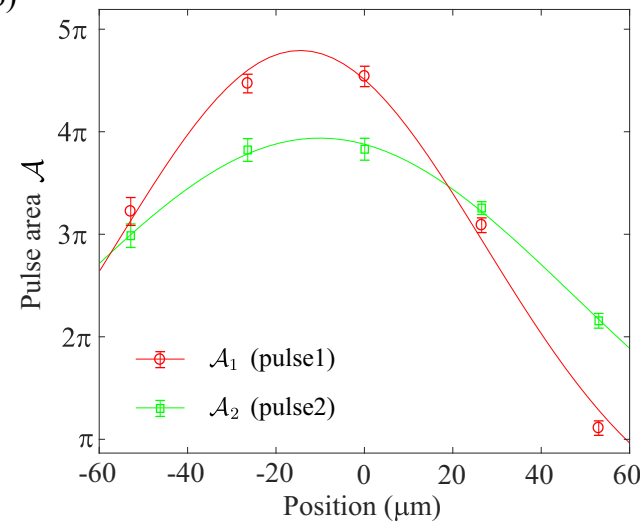

(c)

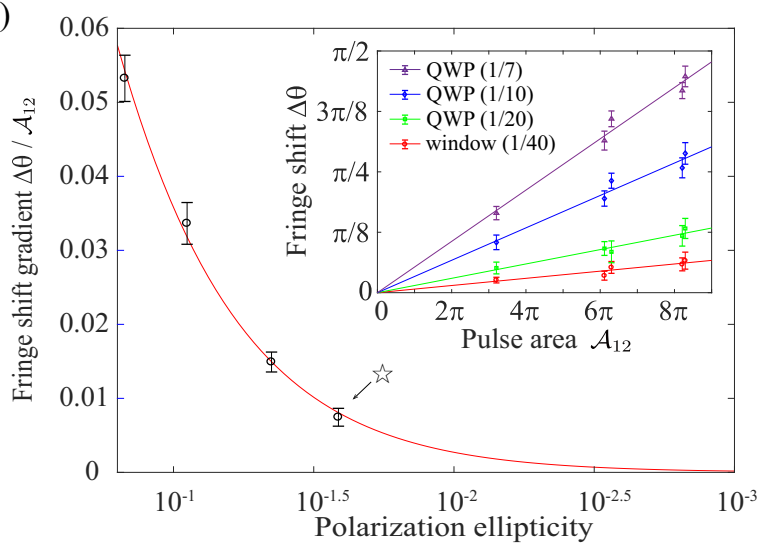

FIG. 3. (a) The $|1\rangle$ state probability of the qubit, prepared in the $|0\rangle$ state followed by the $U_{\hat{x}}$ operation. Each plot shows the probability measured from each single atom at positions $x_{i}$ for various relative polarization angles $\theta$. Black dots are the measurements and the red lines are their fits to a sinusoidal function. (b) Pulse areas measured by Rabi oscillation at five trap sites, respectively, for pulse 1 (red circles) and pulse 2 (green squares), and their fits to a Gaussian function (red and green lines, respectively). (c) Gradient of fringe shift vs the polarization ellipticity for four different values of polarization ellipticity of the pulses: $1 / 40$ (induced by the vacuum window), 1/20,1/10, and 1/7 (varied by a quarter waveplate). The polarization ellipticity induced in the experiment is indicated by the star. The inset shows the fringe shifts $\Delta \theta$ as a function of the pulse area for the four values of polarization ellipticity.

with $n_{x}^{\prime}(T, \mathcal{A})=\cos \left[\varphi_{\hat{n}}+\varphi_{0}(\mathcal{A})\right]$ and $n_{y}^{\prime}(T, \mathcal{A})=\sin \left[\varphi_{\hat{n}}+\right.$ $\left.\varphi_{0}(\mathcal{A})\right]$. Ramsey phase differences $\Delta \varphi_{0}=\varphi_{0}-\left\langle\varphi_{0}\right\rangle$ are shown in Fig. 4, with the Ramsey fringe of each atom in the inset. The Ramsey fringes in the insets exhibit the same phase shift for all the atom positions regardless of the pulse areas, demonstrating the robustness in $\hat{n}$. In other words, all the values of $\Delta \varphi_{0}$ are zero within $95 \%$ confidence intervals among the atom positions. The mean value of the confidence interval radius for all atom positions is $0.016 \pi$, while the standard deviation of the pulse area among all the gates is $1.45 \pi$. So the robustness of the rotation axis against the laser intensity is given within their ratio, i.e., $\delta \varphi_{0} / \delta \mathcal{A}<1.1 \%$.

Now we turn our attention to the numerical estimation of the fidelity and robustness of the given Berryphase gates (for an ideal case without SPAM errors). The Lindblad master equation is used to calculate the amplitude and phase of the transition between the ground hyperfine states $\left|5 S_{1 / 2}, F=2, m_{F}\right\rangle$ and $\left|5 S_{1 / 2}, F=1, m_{F}\right\rangle$, via $\left|5 P_{1 / 2}, m_{J}= \pm 1 / 2\right\rangle\left|I=3 / 2, m_{I}=\mp 1 / 2\right\rangle$ and $\mid 5 P_{1 / 2}, m_{J}=$ $\pm 1 / 2\rangle\left|I=3 / 2, m_{I}= \pm 1 / 2\right\rangle$, in the presence of the offresonant coupling to $\left|5 P_{3 / 2}\right\rangle$ and spontaneous decay. The gate fidelity [31] of $U_{\text {gate }}$ is defined by

$$
\mathcal{F}=\left|\left\langle\psi_{\text {in }}\left|U_{\text {ideal }}^{\dagger} U_{\text {gate }}\right| \psi_{\text {in }}\right\rangle\right|^{2},
$$

where $U_{\text {ideal }}=U_{\hat{x}}(\pi)$ and $U_{\text {gate }}=U\left(\theta_{2}-\theta_{1}=\pi / 2\right)$, averaged over the set of input states, i.e., $\left|\psi_{\text {in }}\right\rangle \in\{|0\rangle,|1\rangle,(|0\rangle+$ $|1\rangle) / \sqrt{2},(|0\rangle+i|1\rangle) / \sqrt{2}\}$. The contributing experimental parameters are the spectral width (FWHM) of the pulses $\Delta \omega$,

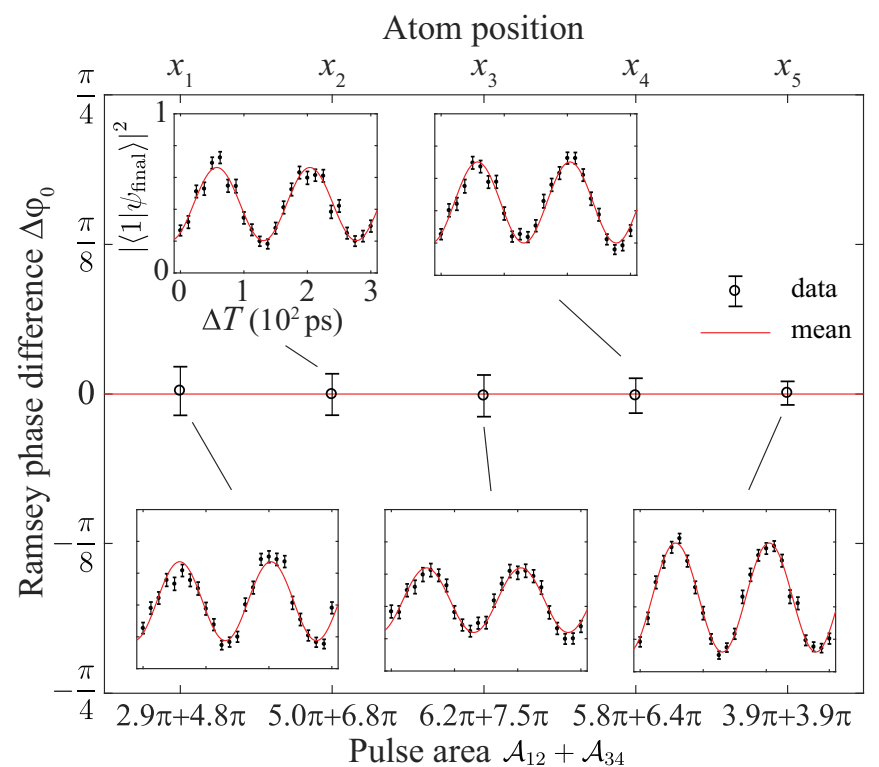

FIG. 4. Ramsey phase difference $\Delta \varphi_{0}$, which is defined as the deviation of the Ramsey phase at each atom from the mean value of it. As in the inset graphs, the Ramsey fringe at each atom position (and each corresponding total pulse area) is measured by the transition probability from $|0\rangle$ to $|1\rangle$ after applying $U_{\hat{x}}(\pi / 2)$ (pulse 1 and pulse 2 ) and $U_{\hat{n}}(\pi / 2)$ (pulse 3 and pulse 4 ) with various interpair time delays $T$, consecutively. Fitted values of $\Delta \varphi_{0}$ are plotted with the error bars indicating the $95 \%$ confidence intervals. 

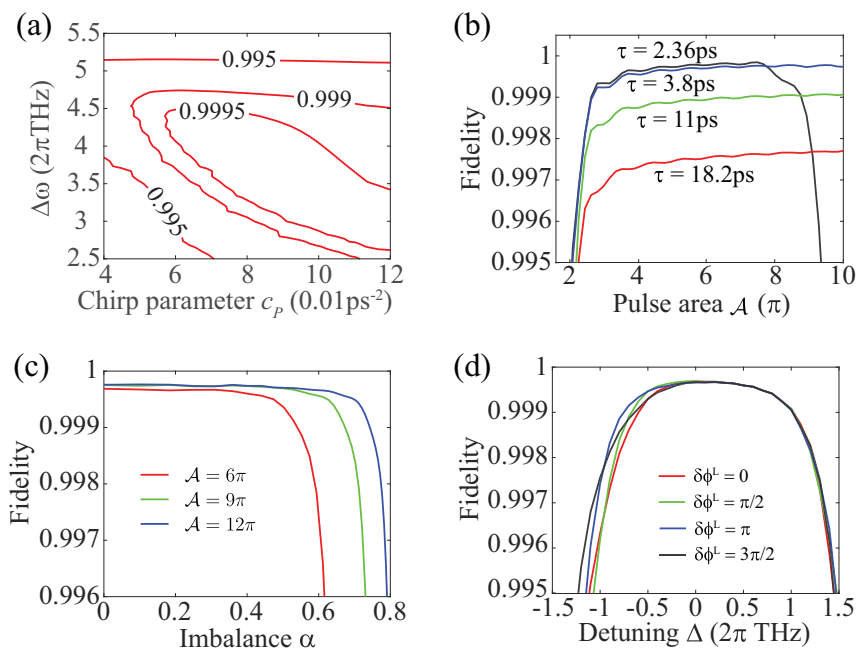

FIG. 5. Calculated fidelity $\mathcal{F}$, for various laser parameters (either varied or otherwise fixed at $\Delta \omega=2 \pi \times 4 \mathrm{THz}, c_{p}=0.072 \mathrm{ps}^{-2}$, $\mathcal{A}=6 \pi, \tau=4 \Delta t_{p}, \alpha=0, \Delta=0$, and $\Delta \phi^{L}=0$ ): (a) $\mathcal{F}\left(c_{p}, \Delta \omega\right)$ as a function of the chirp parameter $c_{p}$ and the spectral width $\Delta \omega$; (b) $\mathcal{F}(\mathcal{A} ; \tau)$ as a function of the pulse area $\mathcal{A}$ for time delays $\tau=18.2$ ps (red), 11 ps (green), 3.8 ps (blue), and 2.36 ps (black); (c) $\mathcal{F}(\alpha ; \mathcal{A})$ as a function of the amplitude imbalance $\alpha$ for pulse areas $\mathcal{A}=6 \pi$ (red), $9 \pi$ (green), and $12 \pi$ (blue); and (d) $\mathcal{F}\left(\Delta ; \delta \phi^{L}\right)$ as function of the detuning $\Delta$ for relative phases $\delta \phi^{L}=0$ (red), $\pi / 2$ (green), $\pi$ (blue), and $3 \pi / 2$ (black).

the chirp parameter $c_{p}$, the pulse area $\mathcal{A}=\mathcal{A}_{1}+\mathcal{A}_{2}$, the time delay $\tau$, the amplitude imbalance of the two pulses $\alpha=$ $\left(\mathcal{A}_{2}-\mathcal{A}_{1}\right) /\left(\mathcal{A}_{2}+\mathcal{A}_{1}\right)$, the frequency detuning $\Delta=\omega_{1}-$ $\omega_{0}=\omega_{2}-\omega_{0}$, and the relative phase between the laser pulses $\delta \phi^{L}=\phi_{2}^{L}-\phi_{1}^{L}$, i.e., $\mathcal{F}=f\left(\Delta \omega, c_{p}, \mathcal{A}, \tau, \alpha, \Delta, \delta \phi^{L}\right)$.

Figure 5 shows the numerical calculation. The contour plot of the fidelity $\mathcal{F}\left(c_{p}, \Delta \omega\right)$ in Fig. 5(a) shows the high-fidelity region around $c_{p} \approx 0.072 \mathrm{ps}^{-2}$ and $\Delta \omega \approx 2 \pi \times 4 \mathrm{THz}$. The spectral width is upper bounded by the leakage $D_{2}$ transition to $5 P_{3 / 2}$ and lower bounded by the insufficient spectral width (smaller than required by the chirp) that corresponds to the spectral width ranging from about $2 \pi \times 3 \mathrm{THz}$ to $2 \pi \times 4 \mathrm{THz}$ in our experiment. To investigate the dependence of the fidelity on the pulse area, amplitude imbalance, and frequency detuning, respectively, in the rest of Fig. 5, we choose $\Delta \omega=2 \pi \times 4 \mathrm{THz}$ and $c_{p}=0.072 \mathrm{ps}^{-2}$. First, in Fig. 5(b), the fidelity $\mathcal{F}(\mathcal{A})$ is calculated as a function of the pulse area $\mathcal{A}$ for various time delays $\tau$. The result exhibits a nearly flat high-fidelity region within a wide range of pulse area, e.g., between $3 \pi$ and $8 \pi$ for a 2.36 -ps short pulse. Thus, the Berry-phase gates are robust against the pulse area (or the laser power fluctuation). This $\mathcal{A}$-robust region $(\delta \mathcal{F} / \delta \mathcal{A} \approx 0$ ) is lower bounded by nonadiabaticity and upper bounded by the interference between temporally close two pulses. Next the robustness against the amplitude imbalance ( $\alpha$ robustness $\delta \mathcal{F} / \delta \alpha \approx 0$ ) is shown in Fig. 5(c), where a sufficiently large pulse area ensures the $\alpha$ robustness as the adiabatic condition of the chirped RAP breaks down for a weaker pulse of insufficient Rabi frequency. Finally, the robustness against the detuning $\Delta$ and relative laser phase $\delta \phi^{L}$ is shown in Fig. 5(d). The $\Delta$ robustness $(\delta \mathcal{F} / \delta \Delta \approx 0)$ is achieved around zero detuning, regardless of the relative phase $\left(\delta \phi^{L}\right.$ robustness), while the asymmetry between positive and negative detunings stems from the dynamic Stark shift (due to the $D_{2}$ transition) of the $5 S_{1 / 2}$ level. Thus, we expect that the fidelity above 0.999 can be achieved for about half of the laser spectral width in the current experiment.

It is worthwhile to compare the robustness of the proposed scheme with a state-of-the-art result. Wang et al. [32] considered the robustness of their single-qubit Pauli $Z$ gate, which is a composite-pulse dynamic phase gate. Their robustness, obtained as $\delta \Theta=21 \mathrm{mrad} \times(\delta f / f)^{2}$ for a laser-induced Stark shift $f$, estimates the fidelity robustness of $\delta \mathcal{F}=10^{-3}$ over a $7 \%$ change in laser intensity. In comparison, our Berry-phase gate is robust over a $40 \%$ change in laser intensity, around $\mathcal{A}=6 \pi$, within $\delta \mathcal{F}=\delta \Theta^{2} / 4=10^{-3}$, estimated based on measurements in Fig. 3(c).

\section{CONCLUSION}

We have implemented qubit rotations of atomic clock states using Berry phases induced by two linearly polarized chirped pulses in picosecond timescales. The results show, as a characteristic of geometric phases of adiabatic passages, gate-operation robustness against laser parameter errors, which has been hard to achieve in previous nonadiabatic holonomic schemes. Berry-phase gates can offer a fast and robust qubit control not only for atomic systems, but also for solid-state systems of relatively short coherence time.

\section{ACKNOWLEDGMENTS}

The authors thank the anonymous referees for valuable comments and suggestions. This research was supported by Samsung Science and Technology Foundation through Grant No. SSTF-BA1301-12.

\section{APPENDIX: DETAILED DESCRIPTION OF THE FAST BERRY-PHASE GATES}

The proposed Berry-phase gates for the atomic clock states are achieved with two successive chirped optical pulses that are linearly polarized or in equal magnitudes of left and right circular polarizations. Each circular polarization component of the chirped pulses adiabatically drives population transfer between the fine-structure ground and excited levels according to the transition selection rules. The Berry-phase difference between the two driven evolution paths is determined by the relative polarization angle between the two pulses, resulting in robust qubit rotation insensitive to other laser parameters except the polarization. After we briefly review the chirped rapid adiabatic passage, we describe the Berry-phase gates for atomic systems.

\section{Chirped rapid adiabatic passages}

The robust population transfer is implemented by using the chirped rapid adiabatic passage [19]. Let us consider a twolevel system, of the fine-structure states $|g\rangle$ and $|e\rangle$ with energy separation $\hbar \omega_{0}$, interacting with a Gaussian chirped pulse of 
an electric field written in the frequency domain as

$$
E(\omega)=\frac{E_{0}}{2} e^{-\left(\omega-\omega_{L}\right)^{2} / \Delta \omega^{2}-i c_{p}\left(\omega-\omega_{L}\right)^{2} / 2}+\text { c.c. },
$$

where $E_{0}$ is the peak amplitude, $\omega_{L}$ is the laser center frequency, $\Delta \omega$ is the bandwidth, and $c_{p}$ is the chirp parameter [33]. The corresponding time-domain electric field is given by

$$
E(t)=\frac{\mathcal{E}}{2} e^{-t^{2} / \Delta t^{2}-i\left(\Gamma t^{2}+\omega_{L} t+\phi^{L}\right)}+\text { c.c. },
$$

with $\quad \mathcal{E}=E_{0} \sqrt{\Delta \omega / \Delta t}, \quad \Delta t=\sqrt{4 / \Delta \omega^{2}+c_{p}^{2} \Delta \omega^{2}}$, $\Gamma=c_{p} /\left(2 c_{p}{ }^{2}+8 / \Delta \omega^{4}\right)$, and $\phi^{L}=-\tan ^{-1}\left(c_{p} \Delta \omega^{2} / 2\right) / 2$. The Hamiltonian of this interaction is given by

$$
H=\frac{\hbar}{2}\left(\begin{array}{cc}
-\Delta(t) & \Omega(t) \\
\Omega(t) & \Delta(t)
\end{array}\right)
$$

in the interaction picture basis $\left|g^{\prime}\right\rangle=\exp \left[-i \int_{-\infty}^{t}\right.$ $\left.\Delta\left(t^{\prime}\right) / 2 d t^{\prime}\right]|g\rangle$ and $\left|e^{\prime}\right\rangle=\exp \left\{-i\left[-\int_{-\infty}^{t} \Delta\left(t^{\prime}\right) / 2 d t^{\prime}+\omega_{0} t+\right.\right.$ $\left.\left.\phi^{L}\right]\right\}|e\rangle$, where $\Delta(t)=\omega_{0}-\omega_{L}-2 \Gamma t$ is the detuning, $\Omega(t)=-\mu \mathcal{E} e^{-t^{2} / \Delta t^{2}} / \hbar$ is the Rabi frequency, and $\mu$ is the transition dipole moment. The eigenstates of Eq. (A3) are given by

$$
\begin{aligned}
& \left|\epsilon_{+}(t)\right\rangle=\sin \vartheta(t)\left|g^{\prime}\right\rangle+\cos \vartheta(t)\left|e^{\prime}\right\rangle, \\
& \left|\epsilon_{-}(t)\right\rangle=\cos \vartheta(t)\left|g^{\prime}\right\rangle-\sin \vartheta(t)\left|e^{\prime}\right\rangle,
\end{aligned}
$$

with $\vartheta(t)=\tan ^{-1}[\Omega(t) / \Delta(t)] / 2$ [for $\left.0 \leqslant \vartheta(t) \leqslant \pi / 2\right]$. The corresponding eigenenergies are given by

$$
\epsilon_{ \pm}(t)= \pm \frac{\hbar}{2} \sqrt{\Omega^{2}(t)+\Delta^{2}(t)} .
$$

Here, since the detuning $\Delta(t)$ is linearly dependent on time, the eigenstate $\left|\epsilon_{-}(t)\right\rangle\left[\left|\epsilon_{+}(t)\right\rangle\right]$ evolves from $|g\rangle(|e\rangle)$ to $|e\rangle(|g\rangle)$ as time changes from $t=-\infty$ to $\infty$, along the meridian of the Bloch sphere. Thus, the complete population transfer between $|g\rangle$ and $|e\rangle$ is achieved as

$$
\begin{aligned}
& |g\rangle \rightarrow-\exp \left[i\left(\frac{1}{2} \int_{-\infty}^{\infty}\left(\Delta+\sqrt{\Omega^{2}+\Delta^{2}}\right) d t-\omega_{0} t-\phi^{L}\right)\right]|e\rangle, \\
& |e\rangle \rightarrow \exp \left[i\left(-\frac{1}{2} \int_{-\infty}^{\infty}\left(\Delta+\sqrt{\Omega^{2}+\Delta^{2}}\right) d t+\phi^{L}\right)\right]|g\rangle
\end{aligned}
$$

when the adiabatic condition

$$
\frac{|\dot{\Omega} \Delta-\Omega \dot{\Delta}|}{2\left(\Omega^{2}+\Delta^{2}\right)^{3 / 2}}=\frac{\Gamma_{p}|\Omega|\left(2 t^{2} / \Delta t^{2}+1\right)}{\left(|\Omega|^{2}+4 \Gamma^{2} t^{2}\right)^{3 / 2}} \ll 1
$$

is satisfied. The rapid adiabatic passage ensures the robustness against the fluctuation of the laser parameters $E_{0}, \omega_{L}$, and $\phi^{L}$ (amplitude, frequency, and phase).

\section{Description of the fast Berry-phase gates in atomic systems}

In our consideration, the qubit states are the hyperfine states $|0\rangle=\left|S_{1 / 2}, F=I+1 / 2, m_{F}\right\rangle$ and $|1\rangle=\mid S_{1 / 2}, F=$ $\left.I-1 / 2, m_{F}\right\rangle$ of the ground state $|g\rangle=\left|S_{1 / 2}, m_{J}= \pm 1 / 2\right\rangle$ of an alkali-metal atom, while the excited level is $|e\rangle=$ $\left|P_{1 / 2}, m_{J}= \pm 1 / 2\right\rangle$. Berry-phase gates are implemented by successive optical transitions between $|g\rangle$ and $|e\rangle$, which induce the phase gates for the qubit system of $|0\rangle$ and $|1\rangle$ (atomic clock states for $m_{F}=0$ ).

Let us consider two chirped pulses 1 and 2 that are time separated by $\tau$ and propagating along the $\hat{z}$ axis, of which the total electric field is given by

$$
\begin{aligned}
\vec{E}(t) & =\hat{n}_{1} E_{1}(t-\tau / 2)+\hat{n}_{2} E_{2}(t+\tau / 2)+\text { c.c. } \\
& =\left(E_{1}^{+} \hat{R}+E_{1}^{-} \hat{L}\right)+\left(E_{2}^{+} \hat{R}+E_{2}^{-} \hat{L}\right)+\text { c.c. },
\end{aligned}
$$

where $\hat{n}_{j}=\hat{x} \cos \theta_{j}+\hat{y} \sin \theta_{j}(j=1,2)$ are the polarization vectors of the pulse $j$ and $E_{j}^{ \pm}(t)=e^{\mp i \theta_{j}} E_{j}\left(t-(-1)^{j} \tau / 2\right)$ are the corresponding electric field components for circular polarizations $\hat{R}=(\hat{x}+i \hat{y}) / \sqrt{2}$ and $\hat{L}=(\hat{x}-i \hat{y}) / \sqrt{2}$, respectively. The interaction Hamiltonian $H_{\text {int }}=-\vec{\mu} \cdot \vec{E}$ has no dependence on $I$ in the picosecond timescale, so the coupling for each polarization component of each pulse is given as an independent two-level system, i.e.,

$$
\begin{aligned}
& \left\langle P_{1 / 2}, m_{J}^{\prime}, I, m_{I}^{\prime}\left|H_{\mathrm{int}}\right| S_{1 / 2}, m_{J}, I, m_{I}\right\rangle \\
& =\left\langle P_{1 / 2}, m_{J}^{\prime}\left|H_{\mathrm{int}}\right| S_{1 / 2}, m_{J}\right\rangle\left\langle I, m_{I}^{\prime} \mid I, m_{I}\right\rangle \\
& =\sum_{j=1,2}\left(\left\langle P_{1 / 2}, 1 / 2\left|-\vec{\mu} \cdot \hat{R} E_{j}^{+}\right| S_{1 / 2},-1 / 2\right\rangle \delta_{m_{J}^{\prime}, m_{J}+1}\right. \\
& \left.\quad+\left\langle P_{1 / 2},-1 / 2\left|-\vec{\mu} \cdot \hat{L} E_{j}^{-}\right| S_{1 / 2}, 1 / 2\right\rangle \delta_{m_{J}^{\prime}, m_{J}-1}\right) \delta_{m_{I}^{\prime}, m_{I}} .
\end{aligned}
$$

In the fine-structure basis $\left|S_{1 / 2}, F=I \pm 1 / 2, m_{F}\right\rangle=$ $\sum_{m_{J}}= \pm 1 / 2 C_{m_{J}, m_{F}-m_{J}}^{1 / 2, I \pm 1 / 2}\left|S_{1 / 2}, m_{J}\right\rangle\left|I, m_{I}=m_{F}-m_{J}\right\rangle$, the time evolution of the ground-hyperfine-state pair $\left|S_{1 / 2}, F=I \pm 1 / 2, m_{F}\right\rangle$ can be described by the time evolution of the two sets of two-level systems $\left\{\mid S_{1 / 2}, m_{J}=\right.$ $-1 / 2\rangle\left|I, m_{F}-1 / 2\right\rangle$ and $\left.\left|P_{1 / 2}, m_{J}=1 / 2\right\rangle\left|I, m_{F}-1 / 2\right\rangle\right\}$, and $\left\{\left|S_{1 / 2}, 1 / 2\right\rangle\left|I, m_{F}+1 / 2\right\rangle\right.$ and $\left.\left|P_{1 / 2},-1 / 2\right\rangle\left|I, m_{F}+1 / 2\right\rangle\right\}$, for $m_{F}=0, \pm 1$. Note here that $m_{F}= \pm 2$ states do not form a pair of hyperfine states, so we will consider only $m_{F}=0, \pm 1$.

For $t \leqslant 0$ (the first pulse case, $j=1$ ), the time evolution from the initial ground hyperfine state $|g\rangle=\mid S_{1 / 2}, m_{J}=$ $\mp 1 / 2\rangle$ to the excited state $|e\rangle=\left|P_{1 / 2}, m_{J}= \pm 1 / 2\right\rangle$ is a rapid adiabatic passage, as described by Eq. (A6a), when the time separation is long enough to satisfy the adiabatic condition, i.e., $\tau \gg 1 / \Gamma_{p}$. So the initial state $\left|S_{1 / 2}, F=I \pm 1 / 2, m_{F}\right\rangle$ evolves to

$$
\begin{aligned}
& -C_{-1 / 2, m_{F}+1 / 2}^{1 / 2, I, I \pm 1 / 2} \exp \left[i \left(\frac{1}{2} \int_{-\infty}^{0}\left[\Delta_{1}+\sqrt{\left(\Omega_{1}^{+}\right)^{2}+\left(\Delta_{1}\right)^{2}}\right] d t\right.\right. \\
& \left.\left.-\omega_{0} t-\phi_{1}^{L}-\theta_{1}\right)\right]\left|P_{1 / 2}, 1 / 2\right\rangle\left|I, m_{F}+1 / 2\right\rangle, \quad \text { (A10a) } \\
& -C_{1 / 2, m_{F}-1 / 2}^{1 / 2, I, I \pm 1 / 2} \exp \left[i \left(\frac{1}{2} \int_{-\infty}^{0}\left[\Delta_{1}+\sqrt{\left(\Omega_{1}^{-}\right)^{2}+\left(\Delta_{1}\right)^{2}}\right] d t\right.\right. \\
& \left.\left.-\omega_{0} t-\phi_{1}^{L}+\theta_{1}\right)\right]\left|P_{1 / 2},-1 / 2\right\rangle\left|I, m_{F}-1 / 2\right\rangle, \quad \text { (A10b) }
\end{aligned}
$$

where $\quad \Omega_{1}^{+}(t)=\left\langle P_{1 / 2}, 1 / 2|-\vec{\mu} \cdot \hat{R}| S_{1 / 2},-1 / 2\right\rangle\left|E_{1}^{+}(t)\right| / \hbar$, $\Omega_{1}^{-}(t)=\left\langle P_{1 / 2},-1 / 2|-\vec{\mu} \cdot \hat{L}| S_{1 / 2}, 1 / 2\right\rangle\left|E_{1}^{-}(t)\right| / \hbar, \quad$ and $\Delta_{1}(t)=\omega_{0}-\omega_{L}-2 \Gamma(t+\tau / 2)$.

For $t \geqslant 0$ (the second pulse case, $j=2$ ), the subsequent adiabatic passage from $|e\rangle$ back to $|g\rangle$, according to Eq. (A6b) 
in Sec. 1, results in

$$
\begin{aligned}
& C_{-1 / 2, m_{F}+1 / 2}^{1 / 2, I, I \pm 1 / 2} e^{i \phi_{-}}\left|S_{1 / 2},-1 / 2\right\rangle\left|I, m_{F}+1 / 2\right\rangle+C_{1 / 2, m_{F}-1 / 2}^{1 / 2, I \pm 1 / 2} e^{i \phi_{+}}\left|S_{1 / 2}, 1 / 2\right\rangle\left|I, m_{F}-1 / 2\right\rangle \\
& =e^{i\left(\phi_{-}+\phi_{+}\right) / 2} \sum_{k= \pm 1 / 2} C_{-k, m_{F}+k}^{1 / 2, I, I \pm 1 / 2} e^{k i\left(\phi_{-}-\phi_{+}\right)}\left|S_{1 / 2},-k\right\rangle\left|I, m_{F}+k\right\rangle \\
& =e^{i\left(\phi_{-}+\phi_{+}\right) / 2} \sum_{k, l= \pm 1 / 2} C_{-k, m_{F}+k}^{1 / 2, I, I \pm 1 / 2} C_{-k, m_{F}+k}^{1 / 2, I, I+l} e^{k i\left(\phi_{-}-\phi_{+}\right)}\left|S_{1 / 2}, F=I+l, m_{F}\right\rangle \\
& =e^{i\left(\phi_{-}+\phi_{+}\right) / 2} U\left(\phi_{-}-\phi_{+}\right)\left|S_{1 / 2}, F=I \pm 1 / 2, m_{F}\right\rangle,
\end{aligned}
$$

where the total phases, for the \pm polarization components, gained during the two adiabatic evolutions are, respectively,

$$
\begin{aligned}
& \phi_{-}=\frac{1}{2} \int_{-\infty}^{0}\left(\Delta_{1}+\sqrt{\Omega_{1}^{+2}+\Delta_{1}^{2}}\right) d t-\frac{1}{2} \int_{0}^{\infty}\left(\Delta_{2}+\sqrt{\Omega_{2}^{+2}+\Delta_{2}^{2}}\right) d t-\phi_{1}^{L}+\phi_{2}^{L}-\theta_{1}+\theta_{2}, \\
& \phi_{+}=\frac{1}{2} \int_{-\infty}^{0}\left(\Delta_{1}+\sqrt{\Omega_{1}^{-2}+\Delta_{1}^{2}}\right) d t-\frac{1}{2} \int_{0}^{\infty}\left(\Delta_{2}+\sqrt{\Omega_{2}^{-2}+\Delta_{2}^{2}}\right) d t-\phi_{1}^{L}+\phi_{2}^{L}+\theta_{1}-\theta_{2},
\end{aligned}
$$

with $\Omega_{2}^{+}(t)=\left\langle P_{1 / 2}, 1 / 2|-\vec{\mu} \cdot \hat{R}| S_{1 / 2},-1 / 2\right\rangle\left|E_{2}^{+}(t)\right| / \hbar, \Omega_{2}^{-}(t)=\left\langle P_{1 / 2},-1 / 2|-\vec{\mu} \cdot \hat{L}| S_{1 / 2}, 1 / 2\right\rangle\left|E_{2}^{-}(t)\right| / \hbar$, and $\Delta_{2}(t)=\omega_{0}-$ $\omega_{L}-2 \Gamma(t-\tau / 2)$. The resulting unitary operations in Eq. (A11) are rotations given, respectively, by

$$
U(\Theta)=\left(\begin{array}{cc}
\cos \frac{\Theta}{2} & -i \sin \frac{\Theta}{2} \\
-i \sin \frac{\Theta}{2} & \cos \frac{\Theta}{2}
\end{array}\right)=\left(\begin{array}{cc}
\cos \frac{\Theta}{2}-i \frac{1}{2} \sin \frac{\Theta}{2} & \mp i \frac{\sqrt{3}}{2} \sin \frac{\Theta}{2} \\
\mp i \frac{\sqrt{3}}{2} \sin \frac{\Theta}{2} & \cos \frac{\Theta}{2}+i \frac{1}{2} \sin \frac{\Theta}{2}
\end{array}\right) \quad \text { for } m_{F}=0, \pm 1
$$

which correspond to $U_{\hat{x}}(\Theta)$ and $U_{\cos (\pi / 3) \hat{z} \pm \sin (\pi / 3) \hat{x}}(\Theta)$. For reference, the rotations for $m_{F}= \pm 2$ are the identity.

Therefore, the two chirped pulses rotate the ground two-level system $\left|0_{m_{F}}\right\rangle \equiv\left|S_{1 / 2}, F=I+1 / 2, m_{F}\right\rangle$ and $\left|1_{m_{F}}\right\rangle \equiv \mid S_{1 / 2}, F=$ $\left.I-1 / 2, m_{F}\right\rangle$ by inducing the relative phase between them. Note that since $\Omega_{j}^{+}(t)^{2}=\Omega_{j}^{-}(t)^{2}$ is satisfied due to the symmetry between $m_{J}= \pm 1 / 2$ and the linear-polarization condition $\left[\left|E_{j}^{+}(t)\right|=\left|E_{j}^{-}(t)\right|\right]$, the dynamic phases represented by the integrals for $\phi_{+}$and $\phi_{-}$in Eqs. (A12) are the same. The relative phase is therefore given by

$$
\phi_{-}-\phi_{+}=2\left(\theta_{2}-\theta_{1}\right) \text {, }
$$

having no parameter dependence except the polarization difference $\theta_{2}-\theta_{1}$ between the two pulses. This is the difference between the Berry phases generated during the two time evolutions of $\left|S_{1 / 2}, \pm 1 / 2\right\rangle$, and thus the qubit rotation implemented by this geometric phase is robust against laser parameters as long as the adiabatic condition in Eq. (A7) is satisfied.

Now we consider more general cases. First, when the dynamic Stark shift due to the off-resonant excited level $P_{3 / 2}$ is taken into account, the detunings $\Delta_{j}^{ \pm}$for $j=1,2$ are to be replaced by

$$
\begin{aligned}
& \Delta_{j}^{+}(t)=\Delta_{j}(t)+\frac{\left|\left\langle P_{3 / 2}, 1 / 2|\vec{\mu} \cdot \hat{R}| S_{1 / 2},-1 / 2\right\rangle E_{j}^{+}(t)\right|^{2}}{4 \hbar^{2}\left[\omega_{0}+\Delta_{\mathrm{fs}}-\omega_{L}-2 \Gamma\left(t-(-1)^{j} \frac{\tau}{2}\right)\right]}+\frac{\left|\left\langle P_{3 / 2},-3 / 2|\vec{\mu} \cdot \hat{L}| S_{1 / 2},-1 / 2\right\rangle E_{j}^{-}(t)\right|^{2}}{4 \hbar^{2}\left[\omega_{0}+\Delta_{\mathrm{fs}}-\omega_{L}-2 \Gamma\left(t-(-1)^{j} \frac{\tau}{2}\right)\right]}, \\
& \Delta_{j}^{-}(t)=\Delta_{j}(t)+\frac{\left|\left\langle P_{3 / 2}, 3 / 2|\vec{\mu} \cdot \hat{R}| S_{1 / 2}, 1 / 2\right\rangle E_{j}^{+}(t)\right|^{2}}{4 \hbar^{2}\left[\omega_{0}+\Delta_{\mathrm{fs}}-\omega_{L}-2 \Gamma\left(t-(-1)^{j} \frac{\tau}{2}\right)\right]}+\frac{\left|\left\langle P_{3 / 2},-1 / 2|\vec{\mu} \cdot \hat{L}| S_{1 / 2}, 1 / 2\right\rangle E_{j}^{-}(t)\right|^{2}}{4 \hbar^{2}\left[\omega_{0}+\Delta_{\mathrm{fs}}-\omega_{L}-2 \Gamma\left(t-(-1)^{j} \frac{\tau}{2}\right)\right]},
\end{aligned}
$$

where $\Delta_{\mathrm{fs}}$ is the fine-structure splitting of the excited states. However, since the linear-polarization condition guarantees $\Delta_{j}^{+}(t)=$ $\Delta_{j}^{-}(t)$ for both $j=1,2$, the presence of $P_{3 / 2}$ makes no difference in Eq. (A14). Second, when the polarization is not perfect and of nonzero ellipticity $\varepsilon_{j}(j=1,2)$, the condition $\left|E_{j}^{+}(t)\right|=\left|E_{j}^{-}(t)\right|$ is replaced by $\left|E_{j}^{+}(t)\right|^{2} /\left|E_{j}^{-}(t)\right|^{2}=\left(1+\varepsilon_{j}\right)^{2} /\left(1-\varepsilon_{j}\right)^{2}$. In this case, the dynamic phases represented by the integrals for $\phi_{+}$and $\phi_{-}$in Eqs. (A12) are not equal, so the dynamic phase is to be included in the qubit rotation angle, making the gate sensitive to laser-parameter fluctuations.

[1] M. V. Berry, Quantal phase factors accompanying adiabatic changes, Proc. R. Soc. London Ser. A 392, 45 (1984).

[2] K. von Klitzing, G. Dorda, and M. Pepper, New Method for High-Accuracy Determination of the Fine-Structure Constant Based on Quantized Hall Resistance, Phys. Rev. Lett. 45, 494 (1980).
[3] S. A. Werner, R. Colella, A. W. Overhauser, and C. F. Eagen, Observation of the Phase Shift of a Neutron Due to Precession in a Magnetic Field, Phys. Rev. Lett. 35, 1053 (1975).

[4] P. Zanardi and M. Rasetti, Holonomic quantum computation, Phys. Lett. A 264, 94 (1999). 
[5] E. Sjöqvist, Geometric phases in quantum information, J. Quantum Chem. 115, 1311 (2015).

[6] F. Wilczek and A. Zee, Appearance of Gauge Structure in Simple Dynamical Systems, Phys. Rev. Lett. 52, 2111 (1984).

[7] R. G. Unanyan, B. W. Shore, and K. Bergmann, Laser-driven population transfer in four-level atoms: Consequences of nonAbelian geometrical adiabatic phase factors, Phys. Rev. A 59, 2910 (1999).

[8] L. M. Duan, J. I. Cirac, and P. Zoller, Geometric manipulation of trapped ions for quantum computation, Science 292, 1695 (2001).

[9] L. Faoro, J. Siewert, and R. Fazio, Non-Abelian Holonomies, Charge Pumping and Quantum Computation with Josephson Junctions, Phys. Rev. Lett. 90, 028301 (2003).

[10] K. Toyoda, K. Uchida, A. Noguchi, S. Haze, and S. Urabe, Realization of holonomic single-qubit operations, Phys. Rev. A 87, 052307 (2013).

[11] F. Kleißler, A. Lazariev, and S. Arroyo-Camejo, Universal, high-fidelity quantum gates based on superadiabatic, geometric phases on a solid-state spin-qubit at room temperature, npj Quantum Inf. 4, 49 (2018).

[12] T. Yan, B.-J. Liu, K. Xu, C. Song, S. Liu, Z. Zhang, H. Deng, Z. Yan, H. Rong, K. Huang, M.-H. Yung, Y. Chen, and D. Yu, Experimental Realization of Nonadiabatic Shortcut to NonAbelian Geometric Gates, Phys. Rev. Lett. 122, 080501 (2019).

[13] E. Sjöqvist, D. M. Tong, L. Mauritz Andersson, B. Hessmo, M. Johansson, and K. Singh, Non-adiabatic holonomic quantum computation, New J. Phys. 14, 103035 (2012).

[14] A. A. Abdumalikov, J. M. Fink, K. Juliusson, M. Pechal, S. Berger, A. Wallraff, and S. Filipp, Experimental realization of non-Abelian non-adiabatic geometric gates, Nature (London) 496, 482 (2013).

[15] C. Zu, W.-B. Wang, L. He, W.-G. Zhang, C.-Y. Dai, F. Wang, and L.-M. Duan. Experimental realization of universal geometric quantum gates with solid-state spins, Nature (London) 514, 72 (2014).

[16] L. Wang, T. Tu, B. Gong, C. Zhou, and G.-C. Guo, Experimental realization of nonadiabatic universal quantum gates using geometric Landau-Zener-Stückelberg interferometry, Sci. Rep. 6, 19048 (2016).

[17] Y. Sekiguchi, N. Niikura, R. Kuroiwa, H. Kano, and H. Kosaka, Optical holonomic single quantum gates with a geometric spin under a zero field, Nat. Photon. 11, 309 (2017).

[18] S.-B. Zheng, C.-P. Yang, and F. Nori, Comparison of the sensitivity to systematic errors between nonadiabatic non-Abelian geometric gates and their dynamical counterparts, Phys. Rev. A 93, 032313 (2016).
[19] N. V. Vitanov, T. Halfmann, B. W. Shore, and K. Bergmann, Laser-induced population transfer by adiabatic passage techniques, Annu. Rev. Phys. Chem. 52, 763 (2001).

[20] Y. Song, H. G. Lee, H. Kim, H. Jo, and J. Ahn, Subpicosecond $X$ rotations of atomic clock states, Phys. Rev. A 97, 052322 (2018).

[21] M. J. Madsen, D. L. Moehring, P. Maunz, R. N. Kohn, Jr., L.-M. Duan, and C. Monroe, Ultrafast Coherent Excitation of a Trapped Ion Qubit for Fast Gates and Photon Frequency Qubits, Phys. Rev. Lett. 97, 040505 (2006).

[22] W. C. Campbell, J. Mizrahi, Q. Quraishi, C. Senko, D. Hayes, D. Hucul, D. N. Matsukevich, P. Maunz, and C. Monroe, Ultrafast Gates for Single Atomic Qubits, Phys. Rev. Lett. 105, 090502 (2010).

[23] J. Lim, H.-g. Lee, S. Lee, C.-Y. Park, and J. Ahn, Ultrafast Ramsey interferometry to implement cold atomic qubit gates, Sci. Rep. 4, 5867 (2014).

[24] A. Ashkin, Acceleration and Trapping of Particles by Radiation Pressure, Phys. Rev. Lett. 24, 156 (1970).

[25] H. Kim, W. Lee, H.-g. Lee, H. Jo, Y. Song, and J. Ahn, In situ single-atom array synthesis using dynamic holographic optical tweezers, Nat. Commun. 7, 13317 (2016).

[26] S. Kuhr, W. Alt, D. Schrader, I. Dotsenko, Y. Miroshnychenko, W. Rosenfeld, M. Khudaverdyan, V. Gomer, A. Rauschenbeutel, and D. Meschede, Coherence Properties and Quantum State Transportation in an Optical Conveyor Belt, Phys. Rev. Lett. 91, 213002 (2003).

[27] S. Zhdanovich, E. A. Shapiro, M. Shapiro, J. W. Hepburn, and V. Milner, Population Transfer between Two Quantum States by Piecewise Chirping of Femtosecond Pulses: Theory and Experiment, Phys. Rev. Lett. 100, 103004 (2008).

[28] A. Steffen, W. Alt, M. Genske, D. Meschede, C. Robens, and A. Alberti, Note: In situ measurement of vacuum window birefringence by atomic spectroscopy, Rev. Sci. Instrum. 84, 126103 (2013).

[29] S. Brakhane, W. Alt, D. Meschede, C. Robens, G. Moon, and A. Alberti, Note: Ultra-low birefringence dodecagonal vacuum glass cell, Rev. Sci. Instrum. 86, 126108 (2015).

[30] N. Solmeyer, K. Zhu, and D. S. Weiss, Note: Mounting ultrahigh vacuum windows with low stress-induced birefringence, Rev. Sci. Instrum. 82, 066105 (2011).

[31] J. F. Poyatos, J. I. Cirac, and P. Zoller, Complete Characterization of a Quantum Process: The Two-Bit Quantum Gate, Phys. Rev. Lett. 78, 390 (1997).

[32] Y. Wang, A. Kumar, T.-Y. Wu, and D. S. Weiss, Single-qubit gates based on targeted phase shifts in a 3D neutral atom array, Science 352, 1562 (2016).

[33] A. M. Weiner, Ultrafast Optics (Wiley, Hoboken, 2009). 